\title{
Safety and toxicological evaluation of a novel Citrus sudachi extract powder
}

\section{Yasuhiro Shikishima ${ }^{1}$, Orie Yoshinari², Yoshiaki Shiojima ${ }^{2}$, Hiroyoshi Moriyama ${ }^{3}$, Manashi Bagchi ${ }^{3}$, Narendra Deshmukh ${ }^{4}$ and Debasis Bagchi ${ }^{*}$}

${ }^{1}$ Ikeda Yakusou Co., Ltd., 1808-1 Shutsu-nakatsu, Ikeda, Miyoshi, Tokushima 778-0020, Japan; ${ }^{2}$ Ryusendo Co., Ltd.,1-5-3 Nishi-ikebukuro, Toshima-ku, Tokyo 171-0021, Japan; ${ }^{3}$ Dr. Herbs LLC, Concord, CA 94521, USA; ${ }^{4}$ INTOX Pvt Ltd, Pune, India; ${ }^{5}$ Department of Pharmacological and Pharmaceutical Sciences, University of Houston College of Pharmacy, Houston, TX 77204, USA

Corresponding author: Debasis Bagchi, PhD, Pharmacological and Pharmaceutical Sciences, University of Houston College of Pharmacy, Houston, TX 77204, USA.

Submission Date: September 14, 2016, Accepted Date: October 28, 2016, Publication Date: October 30, 2016

Citation: Shikishima Y., Yoshinari O., Shiojima Y., Moriyama H., Bagchi M., Deshmukh N., and Bagchi D. Safety and toxicological evaluation of a novel Citrus sudachi extract powder. Functional Foods in Health and Disease 2016; 6(10):677-690

\begin{abstract}
Background: Citrus sudachi, an evergreen tree primarily found in the prefecture of Tokushima, Japan, is a widely used popular citrus fruit used in cooking and consumed as a juice. Citrus sudachi peels are rich in flavonoids including sudachitin (5,7,4'-trihydroxy-6,8,3'trimethoxyflavone), and exhibit potent antioxidant, antimicrobial and anti-diabetic properties; additionally, several limonoids and their glucosides are found in its seeds. We examined the broad spectrum safety of a novel light yellow to golden yellow Citrus sudachi Extract Powder (CSEP), organic, nutritive from the dried fruit rind (25:1 herbs to extract ratio) containing no less than $1 \%$ sudachitin in various toxicology models in GLP-approved laboratories.
\end{abstract}

Methods: The acute oral toxicity study was conducted in female Sprague-Dawley rats with an up and down procedure. The single dose acute dermal $\mathrm{LD}_{50}$ of CSEP was assessed in both male and female rats. The primary skin irritation toxicity of CSEP was assessed in female New Zealand Albino rabbits in order to determine the potential for CSEP to produce irritation after a single topical application, while primary eye irritation index of CSEP was conducted in female New Zealand Albino rabbits. Ames' bacterial reverse mutation assay was conducted to determine the ability of CSEP to induce reverse mutation at selected histidine loci in five tester strains of Salmonella typhimurium viz. TA1535, TA97a, TA98, TA100, and TA102 in the presence and absence of a metabolic activation system (S9) at the doses of 5000, 1500, 500, 150 
and $50 \mathrm{mg} / \mathrm{plate}$. The mutagenic potential of CSEP was also evaluated in an in vitro mammalian cell gene mutation test using the thymidine kinase gene of L5178 $\mathrm{Tk}+/-3.7 .2 \mathrm{C}$ mouse lymphoma cell line.

Results: The acute oral $\mathrm{LD}_{50}$ of CSEP was found to be greater than $5000 \mathrm{mg} / \mathrm{kg}$ body weight. The single dose acute dermal $\mathrm{LD}_{50}$ of CSEP was found to be greater than $2000 \mathrm{mg} / \mathrm{kg}$ body weight in both male and female rats. In the primary skin irritation test, CSEP was found to be slightly irritating to the skin. The primary dermal irritation index (PDII) calculated for CSEP was found to be 0.8 . In the primary eye irritation test, the maximum mean total score (MMTS) of CSEP was found to be 2.7. Thus, CSEP was classified as minimally irritating to the eye. In both Ames' bacterial reverse mutation assay and in vitro mammalian cell gene mutation test, no mutagenicity was observed.

Conclusion: Overall, these toxicological evaluations demonstrate the broad spectrum safety of CSEP.

Keywords: Citrus sudachi Extract Powder (CSEP); acute oral toxicity; acute dermal toxicity; primary dermal irritation; primary eye irritation; Ames' bacterial reverse mutation assay; in vitro mammalian cell gene mutation assay

\section{INTRODUCTION}

Citrus sudachi is a popular, evergreen tree grown in Japan, with its citrus fruits being extensively used as food flavoring in substitution of lemon or lime. Immature fruits are used in cooking, while squeezed juice is often used as an alternative to vinegar or bitter oranges. Approximately 8000 tons of fruit are grown in Japan per year. Research studies have demonstrated its potent neuroprotective, anticancer, and anti-inflammatory properties. Furthermore, scientists have demonstrated its extensive use in metabolic disorders [1-6]. We have developed a novel light yellow to golden yellow CSEP from the dried fruit rind containing no less than $1 \%$ sudachitin as a unique health food supplement. In this study, we conducted a broad spectrum safety and toxicological assessments in a variety of animals and cell culture models in different in vitro and in vivo toxicology models in GLP-approved laboratories to establish the extensive safety of CSEP.

Citrus sudachi hort. ex. Shirai (family: Rutaceae) is an evergreen tree grown in the prefecture of Tokushima, Japan, while the Sudachi fruit is a small, round, green popular citrus fruit in Japan which is used as food flavoring in place of lemon or lime. It is used with many traditional dishes and drinks including fish, ice cream, and vodka coolers. Citrus sudachi contains some polymethoxyflavones including sudachitin (5,7,4'-trihydroxy-6,8,3'trimethoxyflavone) which demonstrated potent neuroprotective, anticancer and antiinflammatory properties, as well as increased bioavailability of calcium [1-6]. Recent studies have demonstrated that sudachitin inhibited nitric oxide production by suppressing the expression of inducible nitric oxide synthase in lipopolysaccharide-stimulated macrophages. Additionally, sudachitin is a superior anti-inflammatory agent when compared to nobiletin [2,3]. Moreover, sudachitin strongly inhibited cyclic nucleotide phosphodiesterase (PDE) activities 
restricted to the $\mathrm{Ca}^{2+}$-calmodulin-dependent (PDE1) and cyclic AMP-specific phosphodiesterase (PDE4) activities, which affirms the potent anti-inflammatory activity of sudachitin [2]. Tsutsumi et al. (2014) have demonstrated that sudachitin improves glucose and lipid metabolism by increasing mitochondrial biogenesis in the skeletal muscle [2]. Furthermore, sudachitin improved dyslipidemia, as evidenced by reduction in triglyceride and free fatty acid levels, and improved glucose intolerance and insulin resistance. Sudachitin also enhanced energy expenditure and fatty acid $\square$-oxidation by increasing mitochondrial biogenesis and function [13]. Sudachitin also increased Sirt1 and PGC-1 $\square$ expression in the skeletal muscle. Tsutsumi et al. (2014) concluded that sudachitin may improve dyslipidemia and metabolic syndrome by improving energy metabolism [2]. Additionally, sudachitin also induces mitochondrial biogenesis to protect against metabolic disorders [2,4-6]. Several limonoids and their glucosides that have been reported in the seeds of the sudachi fruits are responsible for the benefits of the fruit seeds reported so far [3].

In our laboratories, we developed a novel light yellow to golden yellow Citrus sudachi extract powder (CSEP, organic, nutritive, water soluble) from the dried fruit rind (25:1 herbs to extract ratio) containing no less than $1 \%$ sudachitin. In this study, we conducted a broad spectrum safety and toxicological assessments in a variety of in vitro and in vivo toxicology models in GLP-approved laboratories to establish the safety of CSEP. We conducted acute oral toxicity, acute dermal toxicity, primary dermal irritation, primary eye irritation, Ames' bacterial reverse mutation assay (Ames' test) and in vitro mammalian cell gene mutation tests using the thymidine kinase gene of L5178 TK+/- 3.7.2C mouse lymphoma cell line, which were assessed to determine the broad spectrum safety of CSEP.

Overall, these toxicological evaluations demonstrate the broad spectrum safety of CSEP with no adverse effects.

\section{MATERIALS AND METHODS}

\section{Citrus sudachi Extract Powder}

CSEP is a standardized extract from the dried fruit rind of Citrus sudachi (25:1 herbs to extract ratio) (color: light yellow to golden yellow) containing no less than 1\% sudachitin (organic, nutritive, date of manufacture: Nov 2014, Lot \#141111) was used in this study. The powder is soluble in water, moisture content is $<8.0 \%$ and the shelf-life is 2 years.

\section{METHODS}

Acute oral toxicity, acute dermal toxicity, primary dermal toxicity and primary eye irritation of CSEP were conducted at GLP-approved Eurofins/Product Safety Laboratories (Dayton, NJ, USA) as defined in 21CFR58 by the US Food and Drug Administration (FDA, 1987), and in accordance with the Organization for Economic Cooperation and Development (OECD) guidelines for testing of chemicals (OECD 1998)[7,8]. Acute oral toxicity (protocol P322.RAT) was performed with an up and down procedure in female rats following the US EPA Health Test Guidelines OPPTS 870.1100 (2002) and OECD guidelines for the Testing of Chemicals, Test \#425 (2008) and FDA guidelines, while acute dermal toxicity (protocol P320.UDP) was assessed in both male and female rats following US EPA Health Test Guidelines OPPTS 870.1200 (1998) and OECD guidelines for the Testing of Chemicals, Test \#402 (1987) and FDA guidelines. 
Primary skin irritation (protocol P326) was assessed in rabbits following US EPA Health Test Guidelines OPPTS 870.2500 (1998) and OECD guidelines for the Testing of Chemicals, Test \#404 (2002) and FDA guidelines and primary eye irritation (protocol P324) was assessed in rabbits following US EPA Health Test Guidelines OPPTS 870.2400 (1998) and OECD guidelines for the Testing of Chemicals, Test \#405 (2012) and FDA guidelines [7, 8].

GLP-approved Intox Pvt Ltd (Pune, Maharashtra, India) conducted the Ames' bacterial reverse mutation assay of CSEP according to OECD guideline \#471, bacterial reverse mutation test (OECD guidelines for the testing of chemicals, adopted by council on July 21, 1997), and US DHHS/FDA's guidance for industry - ICH S2(R1), Genotoxicity Testing and Data Interpretation for Pharmaceuticals Intended for Human Use, June 2012. Intox Pvt Ltd also conducted in vitro mammalian cell gene mutation test on CSEP using the thymidine kinase gene of L5178 TK+/- 3.7.2C mouse lymphoma cell line according to OECD guideline \#TG 490 "In vitro Mammalian cell gene mutation test using the Thymidine Kinase (TK) gene", OECD guidelines for testing of chemicals, adopted by the council on July 28, 2015, and as per US FDA Redbook 2000, Chapter IV.C.1.c.

Detailed protocols are provided below in individual toxicological assessments.

\section{ANIMAL EXPERIMENTS}

\section{Acute oral toxicity}

The acute oral toxicity evaluation (Up and Down Procedure) was conducted in rats to determine the potential for CSEP to produce acute oral toxicity from a single dose via the oral route. Three healthy young adult female, nulliparous, and non-pregnant Sprague-Dawley derived, albino rats (age: 10 weeks, initial body weight 174-179 g) were obtained from SAGE Labs (Boyerton, PA, USA). A number was allocated to each rat on receipt and a stainless steel ear tag bearing this number was attached to the rat. Airflow measurements were evaluated regularly and the records were kept on file at Product Safety Labs (Dayton, NJ).

The female rats were singly housed in suspended stainless steel perforated bottom caging, which conforms to the size recommendations in the most recent Guide for the Care and Use of Laboratory Animals (National Research Council, 2011; http:/grants.nih.gov/grants/olaw/Guidefor-the-care-and-use-of-laboratory-animals.pdf). Each cage was identified with a cage card indicating at least the study number, dose level, identification and sex of the animal [9]. Litter paper was placed beneath the cage and was changed at least three times per week. The rats had free access to standard rat chow (Harlan Teklad Global 16\% Purina Rodent Diet 2016) adlibitum, except during fasting. Animals had free access to filtered tap water ad libitum, and were maintained at controlled temperature $\left(19-23^{\circ} \mathrm{C}\right)$, relative humidity (36-56\%), and 12 -h light/dark cycle. The animals were acclimated to laboratory conditions at least 14-15 days prior to initiation of dosing. There were no known contaminants reasonably expected to be found in the food or water at levels which would have interfered with the results of this study. Analyses of the food and water are conducted regularly and the records are kept on the file at Product Safety Labs (Dayton, NJ, USA).

CSEP was administered in sequence to the animals, as described in Table 1. The decision to proceed with the next animal was based on the survival of the previous animal following dosing. Before each dosing, the rats fasted overnight, were examined through the fasting period for 
health, and were then weighed (initial). Individual doses were calculated based on the specific gravity and concentration of the test mixture. An initial limit dose of $5000 \mathrm{mg} / \mathrm{kg}$ body weight was administered as a $40 \% \mathrm{w} / \mathrm{w}$ mixture in distilled water to the stomach one healthy female rat by oral gavage using a stainless steel ball-tipped gavage needle attached to an appropriate syringe. Following administration, each animal was returned to its designated cage and the feed was replaced approximately 3-4 h after dosing. Due to the absence of mortality in these animals, two additional females received the same dose levels simultaneously.

Since these animals survived, no additional animals were tested. All animals were observed for mortality, signs of gross toxicity, and behavioral changes during the first several hours postdosing and at least once daily for 14 days after dosing. Individual body weights were recorded prior to administration and again on days 7 and 14 (termination) following dosing. Necropsies were performed on all animals at terminal sacrifice. The animals were observed for mortality, signs of gross toxicity, and behavioral changes during the first several hours post-dosing, and afterwards at least once a day for 14 days after dosing. Observations included gross evaluation of skin and fur, eyes and mucous membranes, respiratory, circulatory, autonomic and central nervous systems, somatomotor activity, and behavioral patterns. Particular attention was directed to observations of tremors, convulsions, salivation, diarrhea, and coma. All rats were euthanized by $\mathrm{CO}_{2}$ inhalation at the end of the 14-day observation period and gross necropsies were performed on all animals. Tissues and organs of the thoracic and abdominal cavities were examined. Data is shown in Table 1.

\section{Acute dermal toxicity study in male and female rats}

An acute dermal toxicity test was conducted on rats to determine the potential for CSEP to produce toxicity from a single topical application. Ten healthy young adult Sprague-Dawley rats (10-11 weeks old) were used in this test. Five male rats and five nulliparous and non-pregnant female rats (body weight: 327-339 g and 211-231 g, respectively) were received from SAGE Labs (Boyertown, PA) and allowed free access to lab chow (Envigo Teklad Global 16\% Protein Rodent Diet \#2016) and filtered tap water ad libitum. Animals were acclimated to laboratory conditions for 21 days prior to initiation of dosing. The animal room was kept at a controlled temperature $\left(19-23^{\circ} \mathrm{C}\right)$, humidity $(45-56 \%)$, and photoperiod (12 h light/12 h dark).

Individual doses of the CSEP were calculated based on the initial body weights obtained prior to dosing at $2000 \mathrm{mg} / \mathrm{kg}$ of body weight (b.w.). On the day prior to application, the hair was removed by clipping the dorsal area and the trunk. After clipping and prior to application, the animals were examined for health, weighed (initial), and the skin checked for any abnormalities. CSEP test product was moistened with distilled water to achieve a dry paste by preparing a $90 \%$ w/w mixture. CSEP (2000 mg/kg b.w.) was then applied to a 2 inch $\times 3$ inch, 4-ply gauze pad and placed on the animal ( $\sim 10 \%$ of the body surface). The gauze pad and entire trunk of each animal were then wrapped with 3-inch Durapore tape to avoid dislocation of the pad and to minimize loss of the test substance. The rats were then returned to their designated cages. The day of application was measured as Day 0 in the study. After $24 \mathrm{~h}$ of exposure to the test substance, the pads were removed and the test sites were gently cleansed of any residual test substance. Individual body weights of the animals were recorded prior to test substance application (initial) and again on days 7 and 14 (termination). The animals were observed for 
mortality, signs of gross toxicity, and behavioral changes the first several hours after application and at least once daily thereafter for 14 days. Observations included gross evaluation of skin and fur, eyes and mucous membranes, respiratory, circulatory, autonomic and central nervous systems, somatomotor activity, and behavior pattern. Particular attention was directed to observation of tremors, convulsions, salivation, diarrhea, and coma. All rats were euthanized via $\mathrm{CO}_{2}$ inhalation on day 14. Gross necropsies were performed on all animals. Tissues and organs of the thoracic and abdominal cavities were examined.

\section{Primary skin irritation study in female rabbits}

A primary dermal irritation test was conducted on rabbits to determine the potential for CSEP to produce an irritation after a single topical application. Three young adult, nulliparous and nonpregnant female New Zealand albino rabbits from Robinson Services Inc. (Clemmons, NC) were allowed free access to lab chow (Envigo Teklad Global High Fiber Rabbit Diet\#2031. A designated amount of the diet (approximately $150 \mathrm{~g} /$ day) and a Premium Timothy Cube ${ }^{\mathrm{TM}}$ (Ontario Dehy Inc) were available to each rabbit. Filtered tap water was supplied ad libitum. Animals were acclimated to laboratory conditions for a period of 9 days prior to initiation of dosing. The animal room was kept at a controlled temperature $\left(19-23^{\circ} \mathrm{C}\right)$, humidity $(47-58 \%)$, and $12 \mathrm{~h}$ light/12 $\mathrm{h}$ dark cycle.

The route of CSEP administration was through a direct application of test substance to shaved intact skin. This route of administration is standard for assessment of local dermal irritative potential. On the day before application, hair on rabbits was clipped from the dorsal and trunk area. On the day of dosing - but prior to application - the animals were examined for health and the skin checked for any abnormalities. No pre-existing skin irritations were observed.

In order to ensure adequate contact with the skin, CSEP was applied as a dry paste $75 \%$ w/w mixture in distilled water). Preliminary sample preparation assessments conducted by PSL indicated that mixtures in excess of $75 \%$ (i,e., 80-95\%) were too dry to assure adequate skin contact.

Five-tenths of a gram of CSEP (0.67 $\mathrm{g}$ of the prepared test mixture) was placed on a 1-inch $\mathrm{x}$ 1-inch, 4-ply gauze pad and applied to one $6-\mathrm{cm}^{2}$ intact dose site of each animal. The pad and entire trunk of each animal were then wrapped with semi-occlusive 3-inch Micropore tape to avoid dislocation of the pad. Elizabethan collars were placed on each rabbit and they were returned to their designated cages. After 4 hours of exposure to CSEP, the pads and collars were removed and the test sites were gently cleansed with a $3 \%$ soap solution followed by tap water and a clean paper towel to remove any residual test substance.

Individual dose sites were scored according to the Draize scoring system [10] (Table 2) at approximately 30-60 $\mathrm{min}, 24,48$, and $72 \mathrm{hr}$ after patch removal. The classification of irritancy was determined by adding the average erythema and edema scores for the 30-60 min, 24, 48 and $72 \mathrm{hr}$ scoring intervals and dividing by the number of evaluation intervals. The resulting Primary Dermal Irritation Index (PDII) was classified according to the descriptive rating in Table 2.

The animals were also observed for signs of gross toxicity and behavioral changes at least once a day during the test period. Observations included gross evaluation of skin and fur, eyes and mucous membranes, respiratory, circulatory, autonomic and central nervous systems, 
somatomotor activity, and behavior patterns. Particular attention was directed to observation of tremors, convulsions, salivation, diarrhea, and coma.

\section{Primary eye irritation study in female rabbits}

The objective was to determine the potential for CSEP to produce irritation from a single instillation via the ocular route. Three healthy young adult female New Zealand albino rabbits (12-14 weeks; 2,538 - 2,630 gms) from Robinson Services Inc. (Clemmons, NC) were allowed free access to lab chow (Envigo Teklad Global High Fiber Rabbit Diet \#2031) and filtered tap water ad libitum. A designated amount of the diet (approximately 150 gms/day) and a Premium Timothy Cube (Ontario Dehy Inc.) were available to each rabbit. Animals were acclimated to laboratory conditions for a period of 5-19 days prior to initiation of dosing. The animal room was kept at a controlled temperature $\left(20-23^{\circ} \mathrm{C}\right)$, relative humidity $(46-52 \%)$, and $12 \mathrm{~h} \mathrm{light} / 12 \mathrm{~h}$ dark cycle.

Prior to test initiation, both eyes of animals were examined using a white light source and a fluorescein dye procedure. One drop of fluorescein sodium dye was instilled into both eyes of each rabbit. The eyes were rinsed with physiological saline $(0.9 \% \mathrm{NaCl})$ after instillation of the fluorescein and then evaluated for corneal damage using an ultraviolet light source. Prior to test substance instillation, the eyes were re-examined and scored for abnormalities according to the "Scale for Scoring Ocular Lesions" as outlined by Draize et al., 1944 [10]. Three healthy, naïve animals (not previously used) without pre-existing ocular irritation were selected for test.

A systematic analgesic (Buprenorphine SR) was administered to relieve potential discomfort associated with eye irritation which provides therapeutic relief for periods of up to 76 hours. Prior to test substance instillation, $0.1 \mathrm{mg} / \mathrm{kg}$ of body weight of the analgesic was administered to the animals and at appropriate intervals to maintain therapeutic blood levels. CSEP was instilled as received. Prior to instillation, 1-2 drops of ocular anesthetic (Tetracaine Hydrochloride Opthalmic Solution USP, $0.5 \%$ ) were placed into both the treated and controlled eye of each animal. One-tenth of a milliliter (0.065 gms) of CSEP was then instilled as a dry powder into the conjunctival sac of the right eye of each rabbit by pulling the lower lid away from the eyeball. The upper and lower lids were then gently held together from about one second before releasing to minimize the loss of CSEP. The other eye of each rabbit remained untreated with CSEP and served as a control. The rabbits were then returned to their designated cages.

Ocular irritation was evaluated using a white light source in accordance with the Draize method of scoring [10] at 1, 24, 48, and $72 \mathrm{hrs}$ post-instillation. The fluorescein dye evaluation procedure was used in the treated eye at 24 hours to verify the absence of corneal damage. Individual scores were recorded for each animal. In addition to observations of the cornea, iris and conjunctivae, any other observed lesions were also noted. The average score for all rabbits at each scoring period was calculated to improve data interpretation.

The time interval with the highest mean score (Maximum Mean Total Score - MMTS) for all rabbits was used to classify the test substance by the system of Kay and Calandra 1962 [11].

The animals were observed for signs of gross toxicity and behavioral changes at least once a day during the test period. Individual body weights of animals were recorded shortly before instillation of CSEP (initial) and at the completion of testing. 
Once testing was complete, the animals were released for euthanasia and humanely euthanized.

\section{IN VITRO EXPERIMENTS}

\section{Mutagenicity Test: Ames' Bacterial Reverse Mutation Assay}

The bacterial reverse mutation assay of CSEP was performed on five mutant tester strains of Salmonella typhimurium viz. TA1535, TA97a, TA98, TA100, and TA102, both in the presence and absence of an exogenous metabolic activation system (rat liver S9) at the doses of 5000, 1500, 500, 150 and 50 mg/plate (Ames et al., 1977; Maron and Ames, 1983)[12,13].

Analytical grade water was chosen as a vehicle and the test doses were selected following a preliminary solubility/precipitation test and a cytotoxicity test. The pre-incubation method was used. Bacterial cells from the five tester strains were incubated with CSEP in a reaction mixture at $37{ }^{\circ} \mathrm{C}$ for 20 minutes, in presence and absence of S9. The reaction mixture was then mixed with the top agar and plated immediately onto minimal medium and incubated for about $48 \mathrm{~h}$ after which the revertant colonies were counted.

The potential mutagenic activity of CSEP was determined by comparing the number of revertant colonies in treated cultures with the number of the revertant colonies in the control cultures. The reproducibility of results was confirmed by repeating the entire study as a second replicate.

\section{In Vitro Mammalian Cell Gene Mutation Assay Using L5178Y/TK+/- 3.7.2C Mouse Lymphoma Cell Line}

This cell line was recommended by the OECD Guideline and procured from ATCC (Bethesda, $\mathrm{MD})$ and maintained at the in vitro Toxicity and Mutagenicity Section of Intox Pvt Ltd at $196^{\circ} \mathrm{C}$, in liquid nitrogen cylinder (cryocan). In Vitro Mammalian Cell Gene Mutation Assay of CSEP using the Thymidine Kinase gene of L5178Y/TK+/- 3.7.2C Mouse Lymphoma Cell Line.

CSEP was evaluated to determine its ability to induce forward mutations in Thymidine Kinase gene (reporter gene) of L5178Y/TK+/- 3.7.2C Mouse Lymphoma Cell Line in the presence and absence of metabolic activation (S9). Based on the results obtained in preliminary tests conducted by microwell method to assess the solubility, precipitation, $\mathrm{pH}$ and cytotoxicity of CSEP, L5178Y/TK+/- 3.7.2C Mouse Lymphoma Cells in suspension were exposed to the test item at the concentrations of $2500,1250,625$ and $312.5 \square \mathrm{g} / \mathrm{mL}$, both in presence (about $3 \mathrm{hr}$ exposure) and absence (about 3 and $24 \mathrm{hr}$ exposure) of an exogenous source of metabolic activation. Liver S9, induced in rats by phenobarbitone and $\square$-naphthoflavone, was used as the metabolic activation system. The exposed cells were sub-cultured to determine cytotoxicity and to allow phenotypic expression. Following phenotypic expression, mutant frequency was determined by seeding known number of cells in medium containing the selective agent to detect mutant colonies, and in medium without selective agent to determine the cloning efficiency (viability) and their frequencies were compared with that in vehicle control group. Concurrent positive control groups were also included in the experiment. The entire study was carried out as Experiment No. 1 ( $3 \mathrm{hr}$ exposure without metabolic activation), Experiment No. 2 (3 hr exposure with metabolic activation), and Experiment No. 3 ( $24 \mathrm{hr}$ exposure without metabolic activation). 


\section{Statistical Analyses}

Eurofins/Product Safety Laboratories (Dayton, NJ) and Intox Pvt Ltd. (Pune, Maharashtra, India) performed statistical analysis of all data collected during the in-life phase of the study, in addition organ weight data. Dupont Haskell Laboratory provided analysis of clinical pathology results to Product Safety Laboratories. Data was analyzed using Bartlett's test, ANOVA, and Dunnett's t-test. All values are reported as mean \pm SD. Statistical significance was set at $\mathrm{p}<$ 0.05. Student's t test was employed for comparing the data generated during and at termination of the recovery period and for comparing food consumption data and urinalysis parameters between control and high dose groups. The variance was evaluated at 5\% level of significance. All statistical analyses were performed using validated statistical programs [14-18].

\section{RESULTS}

The objectives of this study were to determine the safety profile of CSEP. Acute oral toxicity, acute dermal toxicity, primary dermal irritation, primary eye irritation, Ames' bacterial reverse mutation assay, and in vitro mammalian cell gene mutation assay using L5178Y/TK+/- 3.7.2C mouse lymphoma cell line were conducted.

\section{Acute oral toxicity in female rats}

A single oral dose of CSEP was given to female Sprague-Dawley rats to assess its acute toxicity following the Up and Down procedure. CSEP, at the limit dose of level of $5000 \mathrm{mg} / \mathrm{kg}$ body weight, did not cause any mortality and did not demonstrate any signs of gross toxicity, adverse pharmacologic effects, or abnormal behavior in the treated female rats following dosing and during the observation period of 14 days thereafter. All animals survived, gained normal body weight, and appeared active and healthy during the study. No gross abnormalities or pathological alterations were noted for any of the rats when necropsied at the end of the 14-day observation period (Table 1). Based on these results and under the conditions of this study, acute oral $\mathrm{LD}_{50}$ of CSEP was found to be greater than $5000 \mathrm{mg} / \mathrm{kg}$ of body weight in female rats.

Table 1: Acute oral toxicity and observations

\begin{tabular}{|l|l|l|l|l|l|}
\hline \multirow{2}{*}{$\begin{array}{l}\text { Dose level } \\
(\mathbf{m g} / \mathbf{k g})\end{array}$} & \multicolumn{2}{|l|}{ Body weight $(\mathbf{g})$} & $\begin{array}{l}\text { Cage-side } \\
\text { observations } \\
\text { (days 0-14) }\end{array}$ & $\begin{array}{l}\text { Necropsy } \\
\text { observations } \\
\text { (all tissues) }\end{array}$ \\
\cline { 2 - 3 } & Initial & Day 7 & Day 14 & & \\
\hline 5000 & 174 & 217 & 227 & $\begin{array}{l}\text { Active and } \\
\text { healthy }\end{array}$ & $\begin{array}{l}\text { No gross } \\
\text { abnormalities }\end{array}$ \\
\hline 5000 & 174 & 211 & 230 & $\begin{array}{l}\text { Active and } \\
\text { healthy }\end{array}$ & $\begin{array}{l}\text { No gross } \\
\text { abnormalities }\end{array}$ \\
\hline 5000 & 179 & 222 & 226 & $\begin{array}{l}\text { Active and } \\
\text { healthy }\end{array}$ & $\begin{array}{l}\text { No gross } \\
\text { abnormalities }\end{array}$ \\
\hline
\end{tabular}

Female Sprague-Dawley rats were administered a single oral dose of $5000 \mathrm{mg} / \mathrm{kg}$ body weight p.o. of Citrus sudachi Extract Powder in water and meticulously observed over a period of 14 days. 


\section{Acute dermal toxicity study in male and female rats}

An acute dermal toxicity test was conducted on male and female Sprague-Dawley rats to determine the potential for CSEP to produce toxicity from a single topical application. Under the conditions of this study, the single dose acute dermal $\mathrm{LD}_{50}$ of CSEP was found to be greater than $2000 \mathrm{mg} / \mathrm{kg}$ body weight in male and female rats. All animals survived, gained weight, and appeared to be active and healthy during the study. There were no signs of gross toxicity, dermal irritation, adverse clinical effects, or abnormal behavior (Table 2). No gross abnormalities were noted for any of the animals when necropsied at the conclusion of the 14-day observation period.

Table 2. Descriptive rating for primary dermal irritation index (PDII)

\begin{tabular}{|l|l|}
\hline Primary Dermal Irritation Index (PDII) & Classification \\
\hline 0 & Non-irritating \\
\hline$>0-2.0$ & Slightly irritating \\
\hline $2.1-5.0$ & Moderately irritating \\
\hline$>5.0$ & Severely irritating \\
\hline
\end{tabular}

\section{Primary skin irritation study in female rabbits}

The objective of this study was to determine the potential for CSEP to produce irritation from a single topical application to the skin of female New Zealand albino rabbits. All animals appeared active and healthy and gained body weight during the study. Apart from the dermal irritation noted below, there were no signs of gross toxicity, adverse clinical effects, or abnormal behavior. Within 24 hours of patch removal, all three treated sites exhibited very slight erythema and very slight edema. The overall incidence and severity of irritation decreased with time. All animals were free of dermal irritation by $48 \mathrm{~h}$.

The primary dermal irritation index for CSEP is 0.8 (Table 3). Thus, under the conditions of the study, CSEP is classified as slightly irritating to the skin.

Table 3. Summary of primary dermal irritation scores in female New Zealand albino rabbits after exposure to Citrus sudachi Extract Powder (CSEP)

\begin{tabular}{|l|l|l|l|l|}
\hline \multirow{2}{*}{ Time Post-Instillation } & \multicolumn{2}{|l|}{ Incidence of Dermal Irritation ${ }^{\text {a }}$} & Total PDI & $\begin{array}{l}\text { Primary Dermal } \\
\text { Irritation Index } \\
\text { (PDII) }\end{array}$ \\
\cline { 2 - 4 } & Erythema & Edema & $\begin{array}{l}\text { Mean } \\
\text { Score }\end{array}$ & \\
\hline $30-60 \mathrm{~min}$ & 0.7 & 0.3 & 1.0 & 0.8 \\
\hline $24 \mathrm{hr}$ & 1.0 & 1.0 & 2.0 & \\
\hline $48 \mathrm{hr}$ & 0 & 0 & 0 & \\
\hline $72 \mathrm{hr}$ & 0 & 0 & 0 & \\
\hline
\end{tabular}

${ }^{a}$ Values represent mean of three animals. ${ }^{\mathrm{b}}$ Primary dermal irritation (PDI) $=$ average erythema \pm average edema. Primary Dermal Irritation Index (PDII) $=($ Sum of PDI at four time points $) / 4$. 


\section{Primary eye irritation study in female rabbits}

A primary eye irritation test was conducted on female New Zealand albino rabbits to determine the potential for CSEP to produce irritation from a single ocular instillation. Neither corneal opacity nor iritis was observed in any treated eye during this study. One hour after the instillation of CSEP, all three treated eyes exhibited minimal conjunctivitis. The overall incidence and severity of irritation decreased with time. All animals were free of ocular irritation by 48 hours.

All animals appeared active and healthy and gained body weight during this study. Apart from the eye irritation noted, all animals appeared active and healthy. There were no other signs of gross toxicity, adverse clinical effects, or abnormal behavior. Under the conditions of this study, the Maximum Mean Total Score of CSEP is 2.7, classifying CSEP to be minimally irritating to the eye (Tables 4 and 5).

Table 4. Incidence of positive effects, severity, and reversibility of ocular irritation

\begin{tabular}{|l|l|l|l|}
\hline \multirow{2}{*}{$\begin{array}{l}\text { Time } \\
\text { Instillation }\end{array}$} & \multicolumn{2}{|c|}{ Incidence of Positive Effects } \\
\cline { 2 - 4 } & Corneal Opacity & Iritis & Conjunctivitis \\
\hline $1 \mathrm{hr}$ & $0 / 3$ & $0 / 3$ & $0 / 3$ \\
\hline $24 \mathrm{hr}$ & $0 / 3$ & $0 / 3$ & $0 / 3$ \\
\hline $48 \mathrm{hr}$ & $0 / 3$ & $0 / 3$ & $0 / 3$ \\
\hline $72 \mathrm{hr}$ & $0 / 3$ & $0 / 3$ & $0 / 3$ \\
\hline
\end{tabular}

Values represent mean of three animals.

Table 5. Ocular irritation and MMTS

\begin{tabular}{|c|c|c|}
\hline Time Post-Instillation & Severity of Irritation - Mean Score & Severity (MMTS) \\
\hline $1 \mathrm{hr}$ & 2.7 & \multirow[t]{4}{*}{2.7} \\
\hline $24 \mathrm{hr}$ & 0.7 & \\
\hline $48 \mathrm{hr}$ & 0.0 & \\
\hline $72 \mathrm{hr}$ & 0.0 & \\
\hline
\end{tabular}

MMTS (Maximum Mean Total Scores of three animals). See Materials and Methods for details

\section{Mutagenicity Test: Ames' Bacterial Reverse Mutation Assay}

No toxic effect of CSEP was observed in any of the five mutant strains of Salmonella typhirium TA1535, TA97a, TA98, TA100, and TA102 in the presence and absence of a metabolic activation system (S9) at the doses of 5000, 1500, 500, 150 and $50 \mathrm{mg} /$ plate. No biologically relevant increases in revertant colony numbers of any of the five tester strains were observed following treatment with CSEP at any concentration level, in the presence or absence of metabolic activation. CSEP didn't cause gene mutations by base pair changes or frameshifts in the genome of the tester strains used, indicating that CSEP is non-mutagenic. On the basis of the results, interpreted according to OECD 471:1997, the test item CSEP proved to be NonMutagenic for all the tester strains, either in the presence or absence of metabolic activation. 
In Vitro Mammalian Cell Gene Mutation Assay Using L5178Y/TK+/- 3.7.2C Mouse Lymphoma Cell Line

In vitro mammalian cell gene mutation assay of CSEP was carried out using the Thymidine Kinase Gene of L5178Y/TK+/-3.7.2C Mouse Lymphoma Cell line as per OECD and US FDA Redbook 2000 guidelines.

All the cultures, both in presence (about $3 \mathrm{hr}$ exposure) and absence (about 3 and $24 \mathrm{hr}$ exposure) of an exogenous source of metabolic activation, had Mutant Frequencies (MF Mutant per $10^{6}$ cells) that were similar to the average MF of their concurrent vehicle control cultures.

The mutant frequency, cloning efficiency and suspension growth in the vehicle control groups were found to be comparable with the range reported in the literature and in the acceptance criteria as stated in the Mouse Lymphoma Assay (MLA) Workgroup of the International Workshop on Genotoxicity Testing (IWGT).Mutant frequency of concurrent positive control(s) met the acceptance criteria developed by the IWGT Workgroup and demonstrated sensitivity of the assay with and without metabolic activation.

Under the conditions described in this study, it is concluded that CSEP is non-mutagenic when tested in an in vitro mammalian cell gene mutation assay using the Thymidine Kinase gene of L5178Y/TK+/- 3.7.2 Cell line.

\section{DISCUSSION}

Citrus sudachi is a popular evergreen tree; its edible small, round, green citrus lemon-limy fruits are quite popular as food flavoring agent in Japan [1-6]. Citrus sudachi fruits contains sudachitin (5,7,4'-trihydroxy-6,8,3'-trimethoxyflavone), which demonstrated its potent neuroprotective, anticancer and anti-inflammatory properties, in addition to increased bioavailability of calcium $[1,6]$. Sudachitin has been demonstrated to improve glucose and lipid metabolism by increasing mitochondrial biogenesis in the skeletal muscle, as well as improve dyslipidemia, as evidenced by reduction in triglyceride and free fatty acid levels, which opens a new avenue for its wide application in the treatment of metabolic syndrome including obesity and diabetes [2-4]. Furthermore, sudachitin also increased Sirt1 and PGC-1 $\alpha$ expression in the skeletal muscle, which may demonstrate its promise against advancing age and inflammatory responses [3-6]. All these pioneering data motivated us to develop this novel light yellow to golden yellow CSEP from the dried fruit rind (25:1 herbs to extract ratio) containing no less than $1 \%$ sudachitin and explore its extensive safety profile for potential applications in diverse health foods including nutraceutical and functional foods.

The current study evaluated the broad-spectrum safety of novel CSEP in multiple animal models and cultured cells in diverse in vitro and in vivo models. Acute oral toxicity study demonstrated the oral $\mathrm{LD}_{50}$ of CSEP was found to be greater than $5000 \mathrm{mg} / \mathrm{kg}$ body weight in female rats. CSEP didn't induce any signs of adverse pharmacologic effects, gross abnormalities or pathological alterations in the rats when necropsied at the end of the 14-day observation period. This demonstrates the broad spectrum safety of CSEP. The acute dermal toxicity test of CSEP was conducted in male and female Sprague-Dawley rats to produce toxicity from a single topical application. Under the conditions of this study, the single dose acute dermal $\mathrm{LD}_{50}$ of CSEP was found to be greater than $2000 \mathrm{mg} / \mathrm{kg}$ body weight in male and female rats. No toxic 
manifestations were noted in any of these animals when necropsied at the conclusion of the 14day observation period.

Primary skin irritation toxicity of CSEP to produce irritation from a single topical application to the skin of female New Zealand albino rabbits. No gross toxicity of CSEP was observed. The primary dermal irritation index for CSEP is 0.8. Overall, CSEP is classified as slightly irritating to the skin. The primary eye irritation toxicity of CSEP was conducted on female New Zealand albino rabbits. There were no corneal opacity or iritis observed following ocular instillation. One hour after the instillation of CSEP, all three treated eyes exhibited minimal conjunctivitis. The rabbits were free of ocular irritation by 48 hours. The Maximum Mean Total Score of CSEP was found to be 2.7, classifying CSEP to be minimally irritating to the eye (Table 5).

Mutagenic potential of CSEP was assessed in two important models. The bacterial reverse mutation assay of CSEP was performed on five mutant strains of Salmonella typhimurium, via five tester strains of Salmonella typhimurium. TA1535, TA97a, TA98, TA100, and TA102 in the presence and absence of a metabolic activation system (S9) at the doses of 5000, 1500, 500, 150 and $50 \mathrm{mg} / \mathrm{plate}$. CSEP didn't induce gene mutations by base pair changes or frameshifts in the genome of the tester strains used, indicating that CSEP is non-mutagenic. Furthermore, the nonmutagenic potential of CSEP was further reconfirmed by conducting in vitro mammalian cell gene mutation assay using L5178Y/TK+/- 3.7.2C mouse lymphoma cell line. Present study demonstrated that under the conditions of the study CSEP is non-mutagenic. Thus, both of these in vitro assays firmly establish the non-mutagenic potential of CSEP.

\section{CONCLUSION}

Altogether, the data affirms the broad-spectrum safety of CSEP. These results strongly suggest that CSEP can be employed in part of the daily diet, supplementing novel antioxidant bioflavonoids and other essential nutrients, for example in drinks and also dietary supplements, to support good health and attenuate metabolic disorders.

Competing Interests: This study was supported by a research grant from Ikeda Yakusou Co., Ltd., Tokushima, Japan.

Authors' Contributions: All authors contributed to this study. YS is the executive director of Ikeda Yakusou Co., Ltd., Tokushima, Japan. OY and YS are the management team of Ryusendo Co. Ltd., Tokyo, Japan. HM, DB and MB are independent consultants who designed the manuscript based on the independent toxicology studies conducted in professional laboratories. ND is the executive director of INTOX Pvt Ltd, Pune, Maharashtra, India, and directed part of the safety studies.

Acknowledment and Funding: The authors would like to thank Ikeda Yakusou Co., Ltd., Tokushima, Japan, for their funding of this research.

\section{REFERENCES}

1. Yuasa K, Tada K, Harita G, Fujimoto T, Tsukayama M, Tsuji A. Sudachitin, a polymethoxyflavone from Citrus sudachi, suppresses lipopolysaccharide-induced 
inflammatory responses in mouse macrophage-like RAW264 cells. Biosci Biotechnol Biochem 2012; 76(3); 598-600.

2. Tsutumi R, Yoshida T, Nii Y, Okahisa N, Iwata S, Tsukayama M, Hashimoto R, Taniguchi Y, Sakaue H, Hosaka T, Shuto E, Sakai T. Sudachitin, a polymethoxylated flavone, improves glucose and lipid metabolism by increasing mitochondrial biogenesis in skeletal muscle. Nutr Metab (Lond) 2014 Jul 4;11:32. doi: 10.1186/1743-7075-1132. eCollection 2014.

3. Nakagawa H, Duan H, Takaishi Y. Limonoids from Citrus sudachi. Chem Pharm Bull $2001 ; \underline{49}(5) ; 649-651$.

4. Akakabe Y, Sakamoto M, Ikeda Y, Tanaka M. Identification and characterization of volatile components of the Japanese sour citrus fruit Citrus nagatoyuzukichi Tanaka. Biosci Biotechnol Biochem 2008; 72(7); 1965-1968.

5. Nakagawa H, Takaishi Y, Tanaka N, Tsuchiya K, Shibata H, Higuti T. Chemical constituents from the peels of Citrus sudachi. J Nat Prod 2006; 69(8); 1177-1179.

6. Nii Y, Fukuta K, Sakai K, Yamamoto S. Japanese citrus fruit (sudachi) juice is associated with increase bioavailability of calcium from whole small fish and suppressed bone resorption in rats. J Nutr Sci Vitaminol (Tokyo) 2004; 50(3); 177-183.

7. FDA. 1987.Good Laboratory Practices (GLP) for Non-Clinical Laboratory Studies. In: Services. D. o. H. a. H. Washington, DC. US Food and Drug Administration. pp 297310.

8. OECD. 1998. Paris: Committee, C. G. a. M.Principles of Good Laboratory Practice Organization for Economic Cooperation and Development.

9. National Research Council. 2011. Guide for the Care and Use of Laboratory Animals ( $8^{\text {th }}$ Ed.). The National Academies Press, Washingon, DC.

10. Draize JH, Woodward G, Calvery HO. Methods for the study of irritation and toxicity of substances applied topically to the skin and mucous membranes. J Pharmacol Exp Ther 1944; 82; 377-390.

11. Kay JH, Calandra JC. Interpretation of eye irritation tests. J Soc Cos Chem 1962; 13; 281-289.

12. Ames, B.N., McCann, J. and Yamasaki, E. 1977. Methods for the Salmonella Mutagenicity Test. In: Kilbey BJ (Ed.) Handbook of mutagenicity test procedures. Elsevier, Amsterdam. pp 1-17.

13. Maron, D.M., Ames, B.N. 1983. Revised methods for the Salmonella mutagenicity test. Mutation Research 1983; 113; 173-215.

14. Scheffe, H. A method for judging all contrasts in the analysis of variance. Biometrika 40: 87-104.

15. Snedecor, G.W. and Cochran, W. G. 1980. Statistical methods. $7^{\text {th }}$ edition, Iowa State University Press, Ames, Iowa.

16. Bartlett, M.S. 1937. Properties of sufficiency and statistical tests. Proceedings of the Royal Society of London, Series A, 160, 268-282.

17. Dunnett, C.W. 1964. New tables for multiple comparisons with a control. Biometrics 20(3), 482-491.

18. Dunnett, C.W. 1980. Pairwise multiple comparisons in the unequal variance case. Journal of American Statistical Association. 75; 796-800. 